\title{
ROUND ROBIN ON QUANTITATIVE PHASE ANALYSIS
}

\begin{abstract}
Announcement and Call for Expressions of Interest
Initiated and Organized by the IUCr Commission on Powder Diffraction

Aims of the Round Robin

The round Robin will focus on the analysis of powder diffraction data specifically for the derivation of quantitative phase abundance. While the study will focus on the use of laboratory X-ray, synchrotron X-ray and neutron diffraction data, other methods may be used to validate the diffraction results (e.g., FTIR, normative analysis, etc.). The additional methods used will be at the discretion of the participant.
\end{abstract}

\section{General aims}

The general goals of the round robin will include the following:

- To document the methods \& strategies commonly employed in Quantitative Phase Analysis (QPA), especially those involving powder diffraction

- To assess (i) levels of accuracy \& precision, and (ii) lower limits of detection.

- To identify specific problem areas $\&$ develop practical solutions

- To formulate recommended procedures for QPA using diffraction data

- To create a standard set of samples for future reference

Specific aims

The round robin will address the following analytical issues:

Type of analysis:

- diffraction (X-ray/Neutron) vs nondiffraction

- internal std vs external std vs spiking etc standardless methods

Sample features

- representivity \& homogeneity

- particle \& crystallite size

- particle and crystallite statistics

- crystallinity \& surface roughness

- preferred orientation \& microabsorption

Data collection

- type of instrument/geometry

- sample preparation

- data range and wavelength

Data analysis

- integrated intensities vs full-profile Rietveld vs database of observed patterns

- use of constraints and corrections

- software systems \& methods

- complexity of the pattern-peak overlap
Proposed Samples

The samples used in the study will consist of mixtures of major and minor components covering a wide range of analytical complexity.

Simple system

$\alpha-\mathrm{Al} 203+\mathrm{Si}+\mathrm{MgAl} 204$

Preferred orientation

$\mathrm{Mg}(\mathrm{OH}) 2+\alpha-\mathrm{Al} 203+\mathrm{Si}+\mathrm{MgAl} 204$

Crystallinity

$\alpha-\mathrm{Al} 203+\mathrm{Si}+\mathrm{MgAl} 204+$ amorphous

Microabsorption X-ray

$\alpha-\mathrm{Al} 203+\mathrm{Fe} 304+\mathrm{ZrSi} 04+\mathrm{MgAl} 204$

Microabsorption neutron

$\alpha-\mathrm{Al} 203+\mathrm{TiB} 2+\mathrm{MgAl} 204$

Complex system

(i) Granodiorite quartz + feldspar $(2 \times)+$ biotite

(ii) Bauxite ore gibbsite + hematite + kaolinite +etc

(iii) Pharmaceutical system

Types of analyses

The involvement of participants in the round robin can vary depending on the amount of time available to each individual/laboratory. However, because the success of the round robin will depend on the number of results returned, full participation is encouraged.

Possible levels of participation will include the following:

Diffraction data only.

Participant analyses "standard" data sets supplied by the CPD and return details of the analytical procedure plus the results.

Prepared samples.

Participants collect data for analysis from at least two of the samples supplied by the CPD ("simple" and "preferred orientation').

Unprepared samples.

Samples supplied by the CPD are representative of bulk, but may need additional preparation. Participant prepares samples for data collection and analysis.

Because the purpose of the round robin is to assess the methods of quantification, and not identification, the identity of each of the component phases will be supplied. For those participants using Rietveld based methods for the analysis, full structural information for each of the phases will also be supplied. Participants will be asked to return the results using "standard" reporting forms. This standardization should minimize the number of errors likely to occur during transcription of the data. In addition, it is requested that the diffraction data be returned to the CPD as an ASCII format file for re-analysis with a "standard" Rietveld code. 


\section{Timeline}

The following timeline for the round robin is proposed.

In the following schedule, Phase 1 has been largely completed, and we are now at Phase 2.

August/September 1996

Final recommendations for mixtures and protocol Formal approval from CPD

August 96-March 97

Call for expressions of interest

Samples sourced \& prepared for distribution

Collection of standard data

Questionnaire sent to all participants

June 97

Samples and data distributed to participants

December 97

Call-in of results

January-June 98

Analysis of results (including late submissions)

August 98

Preliminary results presented at EPDIC-6

August 99

Presentation of final results at Glasgow IUCr Congress
How to participate

Immediate response is requested.

Potential participants can register their interest in the round robin by sending an E-mail message containing the full contact information to the address given below. Details should include Name, Affiliation, Postal address, Phone, and FAX numbers (including country and area codes) and E-mail address. Respondents will be sent a questionnaire requesting information about which of the samples they wish to analyze plus the amount of sample required (i.e., are they an X-ray or neutron user).

Operating Team

Ian Madsen CSIRO Minerals, Australia

Rod Hill

Edward Groleau CSIRO Minerals, Australia Lachlan Cranswick

Eli Lilly \& Co, USA

Advisory Team Deane Smith (CPD) Penn State University, USA

Jaroslav Fiala Central Res. Inst., Czech Rep.

Contact Details for all Correspondence

Ian Madsen

CSIRO Minerals, PO Box 124, Port Melbourne, Victoria 3207, Australia

Fax: +61 (3) 96463223 ,

E-mail: qpa.rr@minerals.csiro.au 